Session 3532

\title{
Visualization Techniques for Complex Processes in Solid State Engineering
}

\author{
Craig J. Scott and Dana L. Wake \\ Department of Electrical Engineering \\ Morgan State University \\ Baltimore, MD 21239
}

\begin{abstract}
Conventional chalkboard and viewgraph presentation methods sometimes fall short of conveying the abstract concepts that need to be mastered in solid state electronics courses. Visualization techniques using computer-generated animations and threedimensional rendering are explored as instructional delivery methods.
\end{abstract}

\section{Introduction}

Describing the operation of solid state devices relies heavily on graphic and conceptual images. Conventional chalkboard and viewgraph presentation methods sometimes fall short of conveying the abstract concepts that need to be mastered. Computational tools have evolved over the recent past to allow fast rendering of two and three-dimensional graphics on desktop computers and workstations. Precise numerical computations on limitless variations semiconductor device structures are possible.

Methods for the illustration and presentation of these results for non-experts must be both interactive and revealing [1][2][3]. The main goals of visualization are to:

- Convey information,

- Discover new knowledge,

- Identify structure, pattern, anomalies, trends, and relationships.

Most students are eager to use computer software that can simulate real world imagery even if some surrealistic shading is evident. Here, the challenge lies in refocusing this enthusiasm to the subject matter at hand; in this case, solid state engineering. Applications of three dimensional visualization techniques have been applied to circuit analysis [4] to circumvent abstractions in the learning process. Here, the use of computer animation and three-dimensional viewing techniques are investigated as instructional technologies to enhance classroom subject delivery and undergraduate research participation.

\section{Approach}

The basis of the operation of all solid state devices is a set of coupled electromagnetic and statistical equations. The most fundamental equations are listed in table 1. Even at the advanced undergraduate level, these mathematical expressions can be quite intimidating when first introduced. We have adopted two dimensional (2D) and three dimensional (3D) commercial numerical semiconductor device simulators, MEDICI 
and DAVINCI, respectively, from Technology Modeling Associates Inc. (TMA) to handle the arduous task of solving the necessary device equations.

\begin{tabular}{|l|l|}
\hline Equation & Expression \\
\hline Poisson & $\nabla^{2} V=-\frac{\rho}{\varepsilon}$ \\
\hline $\begin{array}{l}\text { Drift- } \\
\text { Diffusion }\end{array}$ & $J_{n}=q v_{n} n E+q D_{n} \nabla n$ \\
$J_{p}=q v_{p} n E-q D_{p} \nabla p$ \\
\hline Continuity & $\frac{\partial n}{\partial t}=\frac{1}{q} \nabla J_{n}+G-R$ \\
& $\frac{\partial p}{\partial t}=-\frac{1}{q} \nabla J_{p}+G-R$ \\
\hline
\end{tabular}

Table 1. Basic semiconductor device equations.

Through this approach, the student can focus on the behavior of the device and not be burdened with mathematical approaches to arrive at a solution. Notwithstanding, the standard one and two-dimensional plots still fall short, if the investigator cannot properly interpret the results. As an alternative, animated movies of the numerical simulations of these devices are used to allow the student to interactively explore the internal workings of these structures. These visualization aids show the behavior and locations of the currents, potentials, and electric fields in the devices as the geometry, material properties, and biasing conditions are changed. Additionally, virtual reality modeling language (VRML) worlds derived from three dimensional numerical simulations of semiconductor devices are employed to let the student interact, in three dimensional space, to investigate the physical behavior of such devices.

\section{Simulation and Visualization Examples}

These instructional technologies have been used in an upper level physical electronics course. The students were given the necessary background and onedimensional closed form equations that relate the terminal currents and voltages to geometric and material parameters. Working in groups, they were asked to simulate and create animations to illustrate various important performance characteristics. In most cases the physical characteristics are plotted as a function of applied bias. The simulations could have been implemented by the instructor. However, this would result in a passive learning mode experience. When the students are actively engaged in the technology as a group, (both device and computer related) they can learn more from each other and appreciate the dynamics of group participation.

\section{The GaAs MESFET Example}

In this exercise the students were charged with developing 2-D illustrations of the potential, electric field, charge carrier, and how this information relates to pinch-off voltage, saturation current, and electric field and potential variations along the channel of the FET. The gate current versus voltage and the I-V family of curves was used as a 
reference to examine what information the $2 \mathrm{D}$ plots were conveying. The results from the student's simulations are shown in figure 1. and figure 2. The 2-D plots were animated in the form of an AVI format movie with the timeline representing the gate voltage changing from pinch-off to open channel.

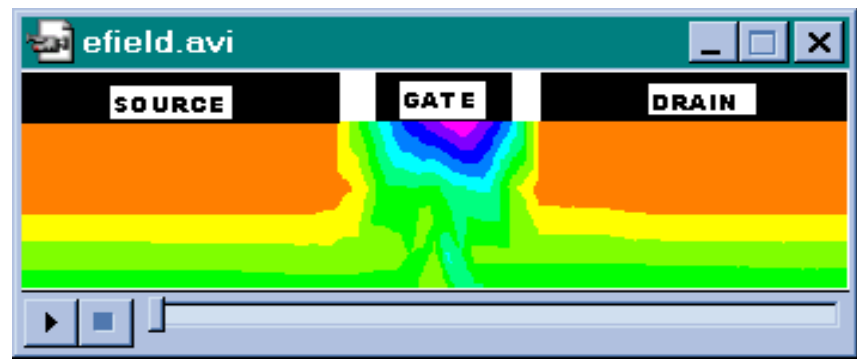

Figure 1. The GaAs MESFET electric field plot for Vgs=0 (open channel).

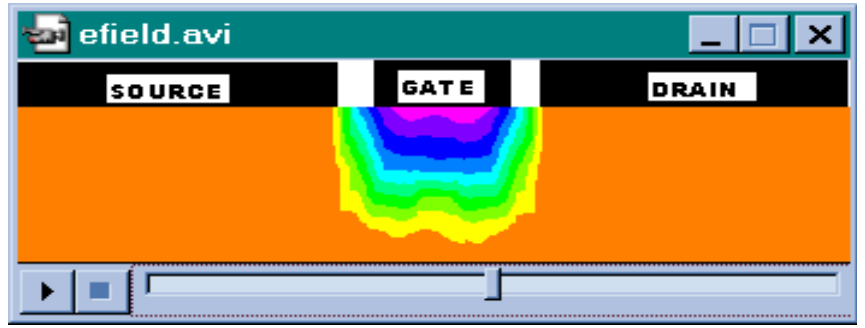

Figure 2. The GaAs MESFET electric field plot for $V g s=V p$. (pinch-off)

The emphasis here was to visualize how the electric field and potential on the gate electrode relates the flow of carriers from the source to the drain. Pinch off occurs when the depletion region edge completely spans the vertical active layer thickness.

The electron current density profile is shown in Figure 3. The carriers leave the source and enter the drain. Most of the carriers are concentrated in the center of the channel. (the dark blue and violet bands.)

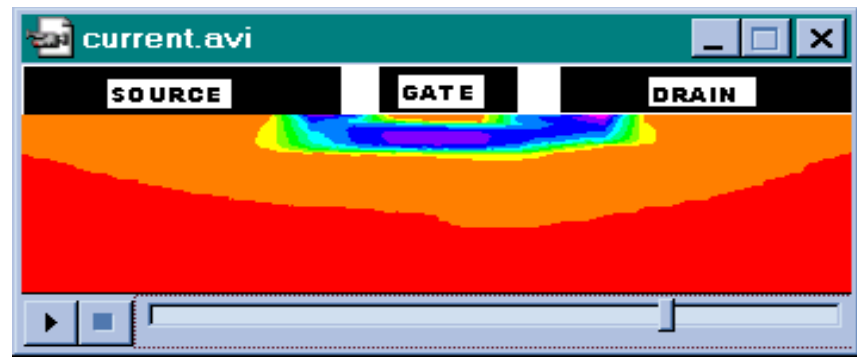

Figure 3. Movie showing the location of carriers in the FET channel under open channel conditions.

\section{The MOSFET VRML Example}

The students pursued this project most enthusiastically because of the three dimensional nature of the visualization. Here the 3-D device simulator was used to generate a spatial mapping of the electron concentration in a MOSFET device. 


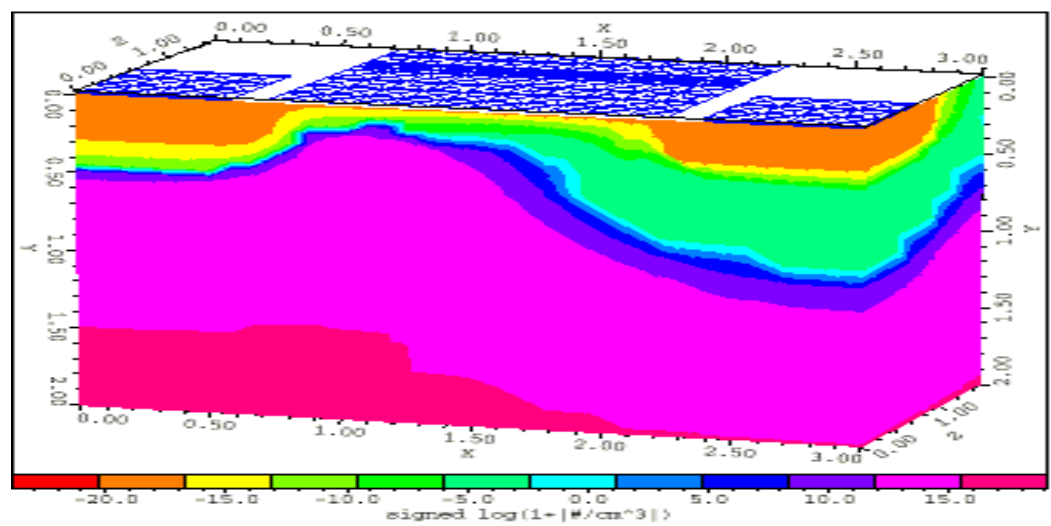

Figure 4. Three dimensional MOSFET graphic showing the location of the inversion layer.

A web browser was created to retrieve the coordinates of the camera or viewpoint and show the surrounding electrons. Volume rendering was simulated using a point cloud graphics primitive. The software used to generate the 3D scene and VRML translation was from the Visualization Toolkit [5]. The user can "walk" around inside and outside the device and examine the electron concentration at any point using the browser.

\section{Discussion}

The above exercises place an emphasis on visualization of complex processes in solid state device engineering. Apart from the conspicuous objective of learning the device behavior from "tweaking" design parameters. One might ask, Where is the design aspect? The design aspect is hidden in the production of the materials by the students. According to the premonitions and observations before and during the assignments the following specifics were accomplished:

- Incorporating small programs for data manipulation.

- Designing an easy to understand data graph.

- Choosing the best technology and approach to implement the illustration.

- Comparison of various approaches and selection of the most viable approach in a group setting.

- Using a time line with benchmarks and milestones.

\section{Conclusions}

Incorporating the latest visualization technology in an upper level undergraduate solid state electronics course presents many opportunities to both the instructor and to the students. This work has the potential to go far beyond the scope of most static approaches routinely used in contemporary courseware in this area. Portraying real space and parametric space, three dimensional concepts through the use of animations and interactive graphics provides a multifaceted learning experience that is both refreshing and "fun". Dazzling the students with rich and interactive visualization tools as compared to unexciting and obscure formulae at the introductory level can stimulate interest. In 
turn, planting these seeds may be the beginning to more rigorous studies at the graduate level.

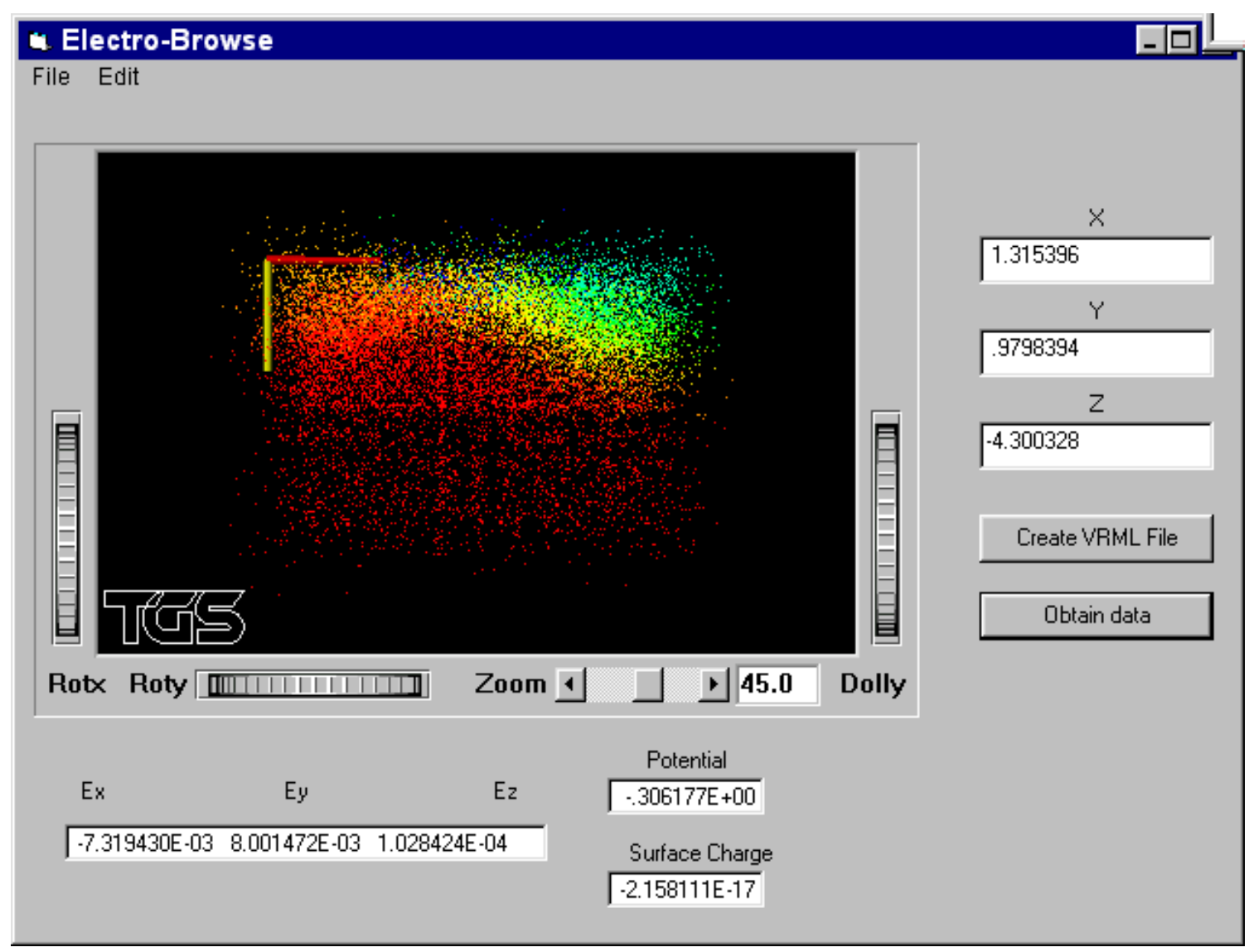

Figure 5. VRML rendition of the MOSFET potential.

\section{References}

[1] Nielson, G.M. "Visualization in Scientific and Engineering Computation. "Computer, September, 1991, pp 58-66.

[2] K.W. Brodlie, L.A. Carpenter, R.A. Earnshaw, J.R. Gallop, R.J. Hubbard, A.M. Mumford, C.D. Osland, P. Quarendon (eds), Scientific Visualization, Techniques and Applications, Springer-Verlag, 1992.

[3] Domik, G., "Education in Scientific Visualization," Proceedings of the IFIP WG 3.2 (Computers in University Education) Working Conference, University of California, Irvine, 1993.

[4] E. R. Doering, "CircuitViz: A New Method for Visualizing the Dynamic Behavior of Electric Circuits," IEEE Transactions on Education, Vol. 39, No. 3, 1996, pp 297-303.

[5] W. Schroder, K. Martin, B. Lorensen, The Visualization Toolkit, Prentice Hall, New Jersey, 1996. 